\title{
A Research on the Weighted Improvement of Dijkstra Algorithm in Optimal Path Calculation
}

\author{
Rongfu Wang ${ }^{1, a}$ \\ ${ }^{1}$ Nanchang Institute of Science \& Technology, Nanchang, Jiangxi, China, 330108
}

Keywords: Safety Engineering; Dijkstra Algorithm; Improvement; Water Inrush Disaster; The Best Water Avoidance Path; Equivalent Path

\begin{abstract}
Dijkstra Algorithm is the shortest path algorithm from one vertex to others, which has solved many shortest path problems. However, in some engineering practice, the shortest path is not the best path, so that it needs to define a weight to illustrate the importance of the two nodes. Based on the best evacuation route when a mine is flooded, the traditional Dijkstra algorithm is improved in this paper. First, this paper interprets the Dijkstra Algorithm before improved, and uses it to calculate the mine shortest path to avoid water, then proposes a improvement method - introducing the concept of equivalent path and analyzing the influence factor and weight of equivalent path, then obtaining the conversion formula of the equivalent path and actual path. Instead of the actual path, the equivalent path can realize the adaptive improvement to the Dijkstra algorithm, so that it can realize the calculation on the best path to avoid water when the water inrush occurs.
\end{abstract}

\section{Introduction}

Dijkstra Algorithm is a typical method for solving the shortest path from the source point to the other points in the network diagram. However, the calculation result is the shortest path from the vertex to the other nodes, which can not be equated with the best hedge path for disaster victims after mine water inrush. Therefore, this paper put forward the improved method of Dijkstra Algorithm, introduced the concept of equivalent path, and obtained the corresponding relation of the shortest path and the optimal path.

\section{The Traditional Process of Dijkstra Algorithm to Solve the Shortest Path}

The following example is used to describe the process of the traditional Dijkstra Algorithm. As shown in figure 1 , it is a case of weighted directed graph, and it be regarded as a simplified topological relation map of mine network. The straight line represents the roadway, A - F is the gateway node, the figure represents the actual length of the roadway.

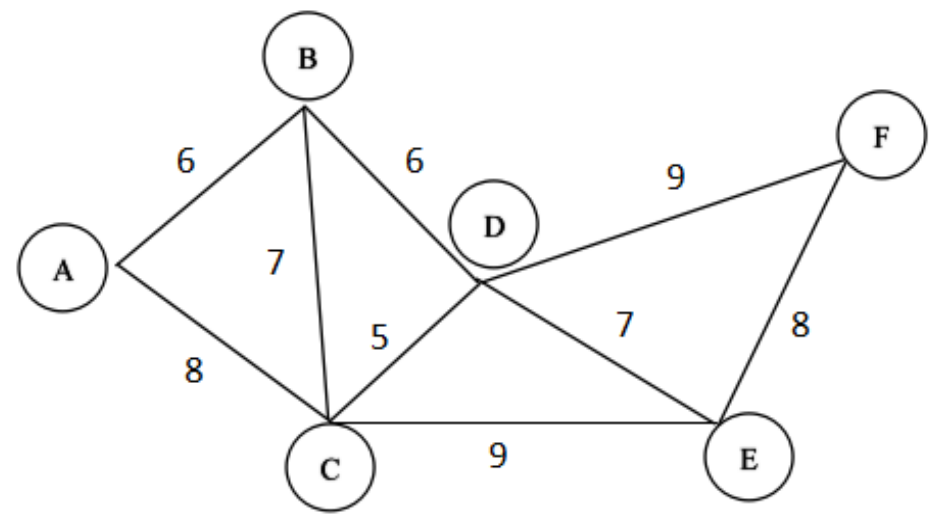

Fig.1 Weighted directed graph example of Dijkstra Algorithm

Suppose A point is the location of mine water inrush and F point is a safe place, with the help of the traditional Dijkstra Algorithm to solve the shortest path from A to F, the specific steps are as 
follows: the nodes in the graph are divided into two groups, the first group, named S, is the vertex set whose shortest path has been solved, and the second group, named $U$, is the residual set. The calculation process is shown in Table 1.

Table 1 The calculation process of the traditional Dijkstra Algorithm

\begin{tabular}{|c|c|c|}
\hline steps & S set & U set \\
\hline 1 & $\begin{array}{c}\text { Selecting } A \text {, here } S=\{A\} \text {, the shortest } \\
\text { path is: } A \rightarrow A=0 \text {, thereafter, searching } \\
\text { outward from } A \text {. }\end{array}$ & $\begin{array}{l}\text { Thereafter, } U=\{B, C, D, E, F\}, A \rightarrow B=6 \text {, } \\
\qquad A \rightarrow C=8, A \rightarrow \text { others }=\infty \text {. }\end{array}$ \\
\hline 2 & $\begin{array}{c}\text { Selecting } B \text {, here } S=\{A, B\} \text {, the shortest } \\
\text { path is: } A \rightarrow B=6 \text {, thereafter, searching } \\
\text { outward from } B \text {. }\end{array}$ & $\begin{array}{c}\text { Thereafter, } \mathrm{U}=\{\mathrm{C}, \mathrm{D}, \mathrm{E}, \mathrm{F}\}, \mathrm{A} \rightarrow \mathrm{B} \rightarrow \mathrm{C}=13 \text { (It } \\
\text { is longer than the path of } \mathrm{A} \rightarrow \mathrm{C} \text {, so it is } \\
\text { abandoned), } \mathrm{A} \rightarrow \mathrm{B} \rightarrow \mathrm{D}=12, \\
\mathrm{~A} \rightarrow \mathrm{B} \rightarrow \text { others }=\infty \text {. }\end{array}$ \\
\hline 3 & $\begin{array}{l}\text { Selecting } D \text {, here } S=\{A, B, D\} \text {, the } \\
\text { shortest path is: } \\
A \rightarrow B \rightarrow D=12 \text {,thereafter, searching } \\
\text { outward from } D \text {. }\end{array}$ & $\begin{array}{l}\text { Thereafter, } \mathrm{U}=\{\mathrm{C}, \mathrm{E}, \mathrm{F}\} \\
\mathrm{A} \rightarrow \mathrm{B} \rightarrow \mathrm{D} \rightarrow \mathrm{C}=17 \text { (It is longer than the path } \\
\text { of } \mathrm{A} \rightarrow \mathrm{C} \text {, so it is abandoned), } \\
\mathrm{A} \rightarrow \mathrm{B} \rightarrow \mathrm{D} \rightarrow \mathrm{E}=20, \mathrm{~A} \rightarrow \mathrm{B} \rightarrow \mathrm{D} \rightarrow \mathrm{F}=21, \\
\mathrm{~A} \rightarrow \mathrm{B} \rightarrow \mathrm{D} \rightarrow \text { others }=\infty\end{array}$ \\
\hline 4 & $\begin{array}{l}\text { Selecting } E \text {, here } S=\{A, B, D, E\} \text {, the } \\
\text { shortest path is: } A \rightarrow B \rightarrow D \rightarrow E=20 \text {, } \\
\text { thereafter, searching outward from } E \text {. }\end{array}$ & $\begin{array}{l}\text { Here } \mathrm{U}=\{\mathrm{C}, \mathrm{F}\}, \mathrm{A} \rightarrow \mathrm{B} \rightarrow \mathrm{D} \rightarrow \mathrm{E} \rightarrow \mathrm{F}=27 \text { (It is } \\
\text { longer than the path of } \mathrm{A} \rightarrow \mathrm{B} \rightarrow \mathrm{D} \rightarrow \mathrm{F}=21 \text {, so } \\
\text { it is abandoned), } \mathrm{A} \rightarrow \mathrm{B} \rightarrow \mathrm{D} \rightarrow \mathrm{E} \rightarrow \mathrm{C}=28 \text { (It is } \\
\text { longer than the path of } \mathrm{A} \rightarrow \mathrm{C}=21 \text {, so it is } \\
\text { abandoned), } \mathrm{A} \rightarrow \mathrm{B} \rightarrow \mathrm{D} \rightarrow \mathrm{E} \rightarrow \text { others }=\infty \text {. }\end{array}$ \\
\hline 5 & $\begin{array}{l}\text { Selecting } C \text { and } F \text {, here } S=\{A, B, C, D \text {, } \\
E, F\} \text {, the shortest path is: } A \rightarrow C=8 \text {, } \\
A \rightarrow B \rightarrow D \rightarrow F=21 \text {, now, the ergodic is } \\
\text { over, stop searching. }\end{array}$ & The U set is empty, so the searching is over. \\
\hline
\end{tabular}

It can be seen from table 1, we can calculate the shortest path from the vertex (point of water) to the various nodes in the roadway with the help of the traditional Dijkstra Algorithm, namely: $\mathrm{A} \rightarrow \mathrm{A}=0, \mathrm{~A} \rightarrow \mathrm{B}=6, \mathrm{~A} \rightarrow \mathrm{C}=8, \mathrm{~A} \rightarrow \mathrm{B} \rightarrow \mathrm{D}=12, \mathrm{~A} \rightarrow \mathrm{B} \rightarrow \mathrm{D} \rightarrow \mathrm{E}=20, \mathrm{~A} \rightarrow \mathrm{B} \rightarrow \mathrm{D} \rightarrow \mathrm{F}=21$. Therefore, in the process evacuating to the safe place $(\mathrm{F}), \mathrm{A} \rightarrow \mathrm{B} \rightarrow \mathrm{D} \rightarrow \mathrm{F}$ is the shortest evacuation route.

\section{The Improvement Method of Dijkstra Algorithm}

In order to calculate the best route of water inrush from disaster (not the shortest path), we introduced the concept of equivalent path. The equivalent path is related to the actual length of the roadway, the type of roadway and the roadway condition, namely:

$$
\operatorname{Lij}=l^{*}(q+t+x)
$$

In the formula, Lij represents the equivalent path from i to $\mathrm{j}$; q represents the type of tunnel weights, dimensionless quantity; $t$ represents the roadway condition, dimensionless quantity; $x$ represents for other factors, dimensionless quantity. The weight value of each influencing factor is [1, 2 ] or $\infty$, if the weight is greater, the evacuation will be more difficult. The roadway types include three types: vertical roadway, inclined roadway and horizontal roadway, and the method to set the weights can refer to table 2 . 
Table 2 The determining method of the roadway type weights

\begin{tabular}{ccc}
\hline \multicolumn{2}{c}{ roadway types and traffic conditions } & roadway type weight \\
\hline \multirow{2}{*}{ vertical roadway } & with transport & 1 \\
& no transport & $\infty$ \\
\hline \multirow{2}{*}{ inclined roadway } & with transport & 1 \\
& no transport & {$[1,2]$ or $\infty$} \\
\hline \multirow{2}{*}{ horizontal roadway, } & with transport & 1 \\
\hline
\end{tabular}

In all the roadway, if there is a traffic tool, the weight can be chosen to be 1 , showing that the efficiency of the roadway is the highest. In the vertical tunnel, if there is no cage hoist, it can not escape from tunnel, so it is infinite. According to the inclination angle of the inclined roadway to choose the weight, if the the angle is greater, the weight will be greater, showing that the escape will be more difficult.

The main influence factors of the roadway condition are height of roadway, water, obstacles and the emergency exit. Its weight setting method can refer to table 3.

Table 3 The determining method of the he parameters in tunnel continuity weight value

\begin{tabular}{|c|c|c|}
\hline retrieval argument & field condition & Value \\
\hline \multirow{4}{*}{ roadway height (a) } & higher than $1.7 \mathrm{~m}$ & 1 \\
\cline { 2 - 3 } & $1.5 \mathrm{~m}-1.7 \mathrm{~m}$ & 1.2 \\
\cline { 2 - 3 } & $1.3 \mathrm{~m}-1.5 \mathrm{~m}$ & 1.4 \\
\cline { 2 - 3 } & $1.1 \mathrm{~m}-1.3 \mathrm{~m}$ & 1.6 \\
\cline { 2 - 3 } & $0.9 \mathrm{~m}-1.1 \mathrm{~m}$ & 1.8 \\
\cline { 2 - 3 } & $0.7 \mathrm{~m}-1.1 \mathrm{~m}$ & 2.0 \\
\cline { 2 - 3 } & lower than $0.7 \mathrm{~m}$ & $\infty$ \\
\hline \multirow{4}{*}{ roadway obstacle (b) } & track, belt conveyor, air door, air bridge & $\infty 1,2]$ \\
\hline \multirow{3}{*}{ roadway water (c) } & higher than $1.5 \mathrm{~m}$ & 2.0 \\
\cline { 2 - 3 } & $1.0 \mathrm{~m}-1.5 \mathrm{~m}$ & 1.8 \\
\cline { 2 - 3 } & $0.5 \mathrm{~m}-1.0 \mathrm{~m}$ & 1.6 \\
\cline { 2 - 3 } & $0.1 \mathrm{~m}-0.5 \mathrm{~m}$ & 1 \\
\cline { 2 - 3 } & lower than $0.1 \mathrm{~m}$ & $\infty$ \\
\hline \multirow{3}{*}{ emergency exit (d) } & yes & 1 \\
\cline { 2 - 3 } & no & $\infty$ \\
\hline
\end{tabular}

According to practical experience, if the roadway height is higher than the average height of the adult male, the pass efficiency is the biggest, the value of a is equal to 1 , if the roadway is too low to pass, the value of a is equal to $\infty$; If there is no obstacles in the roadway, the value of $b$ is equal to 1 , with an increase of one obstacle, the coefficient increased by 0.1 ; If there no water or the water level is lower than the height of the ankle, the value of $\mathrm{c}$ is equal to 1 , if water is higher than the chest, roadway is not out of traffic, the value of $\mathrm{c}$ is equal to $\infty$; If there is no emergency exit, the value of $\mathrm{d}$ is equal to $\infty$, or $\mathrm{d}=1$. Namely, the calculation formula of the weight of the roadway is as follows:

$$
t=a * b * c * d
$$

Bring formula (2) into formula (1), we can get the conversion formula of the equivalent path and the actual path, that is:

$$
\operatorname{Lij}=l^{*}[q+(a * b * c * d)+x]
$$

By formula (3), the equivalent length of each roadway can be obtained. This formula fully considered the influence of actual length, types and the conditions of the roadway, so with the help of the Dijkstra Algorithm, it can the best hedge after mine water inrush, provide the right decision for avoiding disaster, ensure the trapped person get the safety place in the fastest time, thus reducing 
casualties under the mine.

\section{The Improved Dijkstra Algorithm and Its Optimal Path Solution}

Assumption of the roadway type and condition. According to the method in table 1 and table 2 to assume the roadway type and condition, then figure out the equivalent length of roadway, as shown in Table 4.

Table 4 The continuity assumptions of tunnel type and the calculation of the tunnel equivalent length

\begin{tabular}{ccccc}
\hline name & length & type and weight & condition weights & equivalent length \\
\hline A-B & 6 & vertical roadway, $\infty$ & 1.30 & $\infty$ \\
A-C & 8 & inclined roadway, 1.20 & 1.30 & 12.48 \\
B-C & 7 & inclined roadway, 1.10 & 1.40 & 10.78 \\
B-D & 6 & inclined roadway, 1.10 & 1.20 & 7.92 \\
C-D & 5 & horizontal tunnel, 1.10 & 1.40 & 7.70 \\
C-E & 9 & inclined roadway, 1.40 & 1.30 & 16.38 \\
D-E & 7 & inclined roadway, 1.40 & 1.20 & 11.76 \\
D-F & 9 & horizontal tunnel, 1.20 & 1.10 & 11.88 \\
F-E & 8 & inclined roadway, 1.30 & 1.10 & 11.44 \\
\hline
\end{tabular}

The topological relation of the improved roadway. Instead of the actual length of roadway, using the equivalent length to establish the network topological relation of underground roadway, as shown in Figure 2.

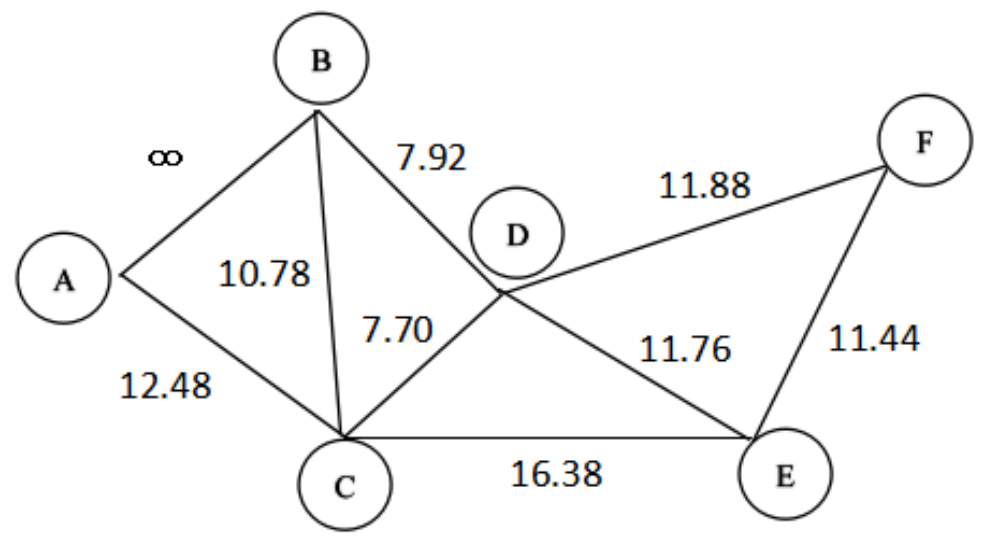

Fig.2 The improved tunnel network topology diagram

The connection of A to B is shaft with no means of transport, therefore, the equivalent length of roadway is marked as $\infty$. In the calculation process, it can be ignored, and the equivalent length of the remaining roadway is calculated according to the method provided by formula (3).

The solution of the optimal path. Based on the traditional Dijkstra Algorithm in Table 1, resolving the improved network topology relationship can figure out the optimal path from the A to the safe location $\mathrm{F}$, namely: $\mathrm{A} \rightarrow \mathrm{C} \rightarrow \mathrm{D} \rightarrow \mathrm{F}$. The total length of the roadway is 32.06, and the shortest path calculated by this path is significantly different from that calculated in Table 1 . The reason calling the $\mathrm{A} \rightarrow \mathrm{C} \rightarrow \mathrm{D} \rightarrow \mathrm{F}$ the best way to escape the disaster is that it added the roadway type and condition in the actual path, and it has fully considered the influence factors of the evacuation of the trapped personnel. Compared with the shortest path, this evacuation route is more scientific to ensure that trapped people reach a safe place in the shortest time. 


\section{Conclusion}

1) This paper demonstrated the limitations of the traditional Dijkstra Algorithm, put forward the shortest path after the mine water inrush may not be the optimal path, therefore, the traditional Dijkstra Algorithm can not be directly used to figure out optimal path after the mine water inrush.

2) This paper put forward the concept of equivalent path and determined the conversion formula of the equivalent path and the actual path, so the traditional Dijkstra Algorithm got improved, which provides a new method for the calculation of the optimal path.

\section{References}

[1] Guanzheng TAN, Huan HE, Aaron Sloman, 2006. Global optimal path planning for mobile robot based onimproved Dijkstra algorithm and ant system algorithm. Journal of Central South University of Technology(English Edition), 01, 80-86.

[2] Vladica Tintor, Jovan Radunovic, 2009. Distributed Dijkstra sparse placement routing algorithm for translucent optical networks. Photonic Network Communications, 03, 181-189.

[3] Viacheslav Chernoy, Mordechai Shalom, Shmuel Zaks, 2010. On the performance of Dijkstra's third self-stabilizing algorithm for mutual exclusion and related algorithms. Distributed Computing,b02,b231-235.

[4] Hui FU, 2013. The Mine Water-inrush and Avoid Disaster Simulation and Algorithm Research and Implementation. China University of Mining and Technology (Beijing), 2013.

[5] Hua WANG, 2014. Improvement of Dijkstra's Algorithm by Using Combinatorial Techniques. Science of Surveying and Mapping, 02, 52-54.

[6] Zuopeng ZHAO, Guojuan SONG, Yuanyuan ZONG, 2015. Research on the Multi-optimal Paths of Coal Mine Floods Based on the D-K Algorithm[J]. Journal of China Coal Society, 02, 397-402. 